\title{
Evaluación de una ontología para el intercambio de información materno-infantil entre instituciones de salud en México
}

\author{
E. V. López Hernandez ${ }^{1}$, Beatriz A. Olivares Zepahua ${ }^{1}$, G. S. Hernández Chan², \\ José L. Sánchez Cervantes ${ }^{2}$ \\ ${ }^{1}$ Instituto Tecnológico de Orizaba, División de Estudios de Posgrado e Investigación, \\ Orizaba, Veracruz, México \\ ${ }^{2}$ Instituto Tecnológico de Mérida, Dirección de Ingeniería Eléctrica y Electrónica, \\ Yucatán, México \\ erwinl@acm.org, bolivares@ito-depi.edu.mx, gandhi.hernandez@itmerida.mx, \\ cervantes82@msn.com
}

Resumen. El proceso de evaluación de una ontología es una tarea complicada, ya que hay pocas metodologías o herramientas que permitan realizar esta actividad de manera automática y fundamentada. Existen diversos enfoques para realizar la evaluación de ontologías dependiendo del tipo específico y sus propósitos. En este artículo se describe el proceso de evaluación de la calidad de la ontología para el intercambio de información materno-infantil mediante la implementación de la aplicación Web OOPS!, la cual a través de un catálogo de errores comunes permite detectar problemas de diseño y modelado de ontologías.

Palabras clave: Ontología, calidad, evaluación, OOPS!

\section{Evaluation of an Ontology for the Exchange of Maternal and Child Information Among Health Institutions in Mexico}

\begin{abstract}
Absract: The process of evaluating ontology is a complicated task, since there are few methodologies or tools that allow this activity to be performed automatically and grounded. There are several approaches to perform the evaluation of ontologies depending on their specific type and purpose. This article describes the process of evaluating the quality of the ontology for the exchange of maternal and child information through the implementation of the OOPS! Web application, which through a catalog of common errors allows to detect design and modeling problems of ontologies.
\end{abstract}

Keyswords: Ontology, quality, evaluation, OOPS! 


\section{Introducción}

El presente trabajo presenta el proceso de diseño, elaboración y evaluación de una ontología orientada al intercambio de información materno-infantil, es decir, de mujeres y niños en periodo perinatal y neonatal en hospitales de México.

El problema que se ha observado gira entorno a la atención tanto de mujeres embarazadas y recién nacidos que son pacientes de hospitales o centros de salud especializados en esta área de la medicina. El problema radica sobre todo en cuanto al manejo de información y el intercambio de la misma. Esto es, se ha notado que existe una diferencia en la forma en que los hospitales almacenan y procesan la información. Esta diferencia, además de retrasar el proceso de atención, y complicar el intercambio de la misma, provoca problemas como falta e inconsistencia de información.

Cabe señalar, que si bien la información relacionada con expedientes médicos es importante, en el caso particular de la atención materno-infantil lo es aún más, ya que implica un vínculo o relación entre la información de la madre y del hijo, relación que por ninguna razón debe perderse o verse afectada por cuestiones de seguridad.

Dada esta situación, la solución propuesta es el diseño de una ontología que, en primera instancia, permita, de alguna forma, estandarizar los diferentes formatos de la información que se maneja en los distintos hospitales, y, como consecuencia de ello, facilite su intercambio. Todo ello con el objetivo de apoyar a las instituciones de salud a disminuir las tasas de mortalidad materno-infantil en México.

En el proceso de elaboración de la ontología se aplicó la metodología Methontology, la cual proporciona actividades, guías y un ciclo de vida que permiten llevar a cabo el desarrollo de la ontología. Sin embargo, Methontology no describe la manera de evaluar la calidad de lo obtenido. Durante el subproceso de validación y evaluación de la ontología es importante contar con herramientas formales que permitan determinar si la ontología construida cumple con los requerimientos específicos, las necesidades, expectativas o si es posible reusar el conocimiento sobre un dominio determinado. Para este proceso se ha utilizado la herramienta OOPS!

\section{Diseño de ontología para el intercambio de información materno-infantil}

Las instituciones públicas de salud en México funcionan bajo el esquema de derechohabiencia, es decir, una persona debe registrarse ante la institución para tener derecho a recibir atención médico-hospitalaria de dicha institución. Sin embargo, todas las instituciones de salud, públicas o privadas, están obligadas a atender los casos de emergencia que se presenten, con independencia de la derechohabiencia del paciente; por supuesto, contar con el expediente clínico del paciente facilita el brindar una atención de calidad ya que permite conocer tanto el historial como la situación actual del paciente más allá de los síntomas de la emergencia. Esto es particularmente importante en el caso de las mujeres embarazadas y los recién nacidos, pues la falta de información relevante en el momento de la atención deriva fácilmente en la muerte. Pese a la importancia de contar con el expediente clínico en el momento de la atención, no es fácil obtenerlo cuando el paciente es derechohabiente de una institución distinta 
a la que brinda la atención, debido a que, aun en el caso de expedientes electrónicos, es común que algunos términos, sobre todo administrativos, difieran entre instituciones.

Por lo anterior, se planteó el diseño de una ontología para el intercambio de información materno-infantil entre los sistemas de instituciones de salud en México. La ontología considera requerimientos legales vigentes en México aplicables a la atención materno-infantil y al intercambio de expedientes médicos tales como Norma Oficial Mexicana NOM-004-SSA3-2012 del expediente clínico, NOM-024-SSA3-2012 Intercambio de información en salud, entre otros. Además se implementó el estándar internacional Health Level Seven (HL7) para minimizar las incompatibilidades entre sistemas de información en salud y la reutilización parcial de la solución semántica SNOMED-CT la cual proporciona una manera estandarizada de representar frases clínicas capturadas por los especialistas.

En la figura 1 se muestran los conceptos sobresalientes obtenidos en el desarrollo de la ontología, se observa que el concepto Persona es el concepto principal de la ontología, ya que cuenta con relaciones con los demás conceptos. Además se visualiza que los otros conceptos no cuentan con relaciones entre ellos, esto se debe a que la ontología está diseñada para consultar información del expediente clínico de mujeres embarazadas y de recién nacido.

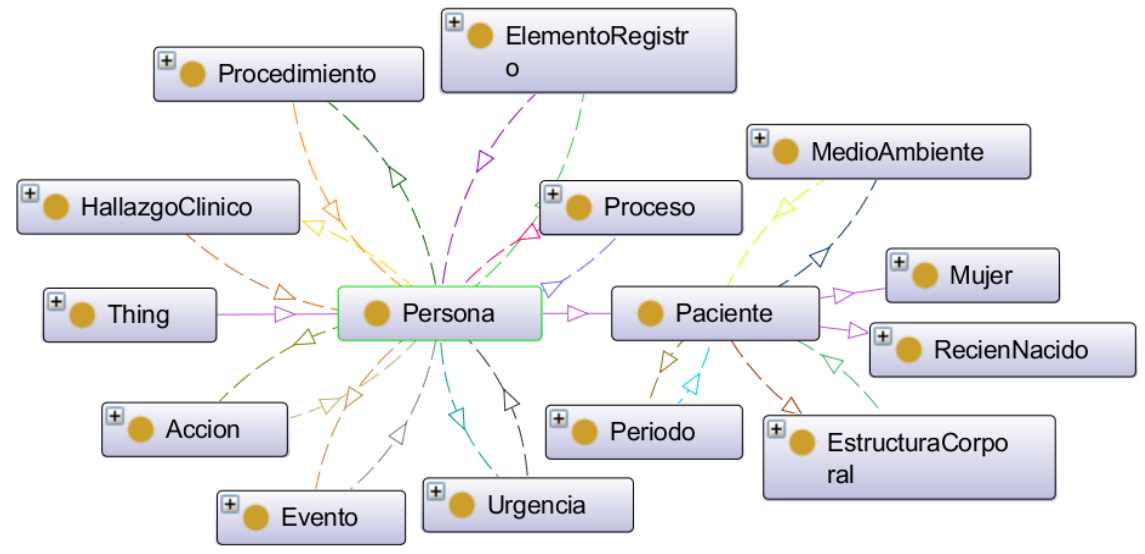

Fig. 1. Diagrama de relaciones binarias.

Para comprobar que la ontología cumple con el objetivo de su desarrollo, se considera contar con una aplicación cliente que envíe la solicitud de información acerca de un paciente en particular y una aplicación proveedor que dé respuesta a la solicitud. En la figura 2 se muestran las tecnologías y herramientas necesarias para que sistemas de salud en México logren compartir expedientes clínicos de mujeres embarazadas y recién nacidos. Cabe hacer notar que el proceso de las aplicaciones soportaría expedientes de todo tipo de pacientes, sin embargo la ontología, por el momento, sólo considera el mapeo de información específicamente relacionada con salud maternoinfantil. 


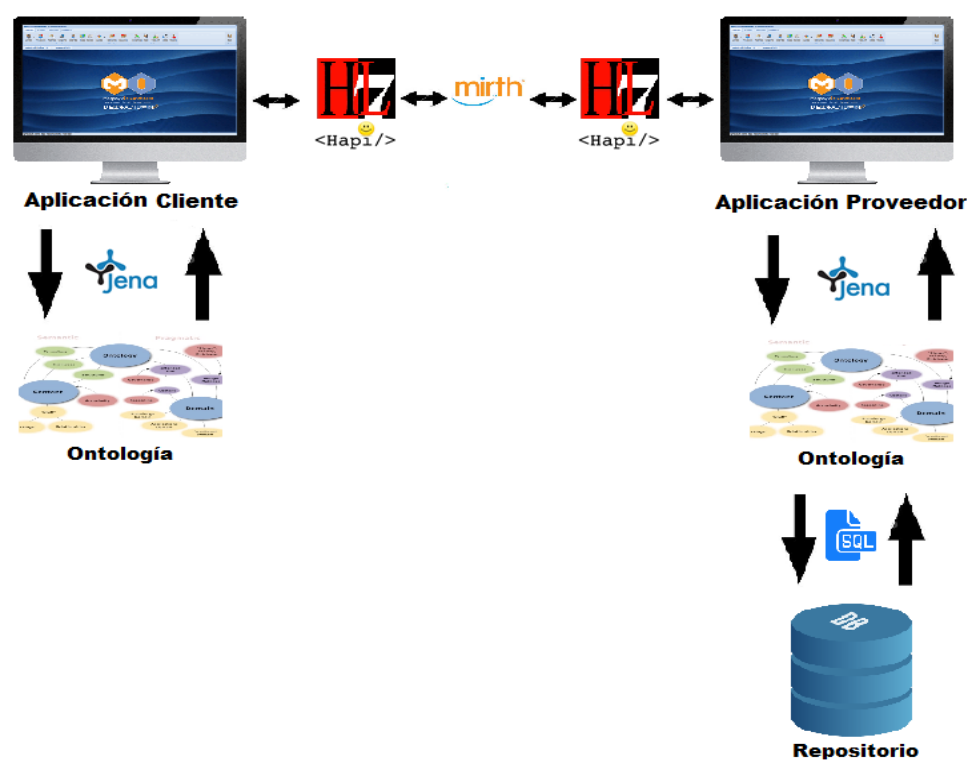

Fig. 2. Esquema de la funcionalidad de la ontología.

Las tecnologías necesarias para el intercambio de información indicadas en la figura anterior son:

- HL7 es un conjunto de estándares que apoyan la práctica clínica y la gestión, prestación y evaluación de los servicios de salud, que se reconocen como los más utilizados en el mundo [1].

- Mirth Connect es un motor de integración de software libre basado en Java, desarrollado específicamente para integraciones sanitarias y que provee de manera nativa el estándar de mensajería HL7 [2].

- Jena es una API Java que se utiliza para construir aplicaciones de Web Semántica y Linked Data. Trabaja con modelos RDFS y OWL (Web Ontology Languaje) para agregar semántica adicional a los datos RDF [3].

- HAPI es una biblioteca y una aplicación enriquecida para editar, validar y transmitir mensajes HL7 [4].

La funcionalidad de la aplicación cliente es la siguiente:

1. Primero es necesario configurar la aplicación cliente, que consiste en crear los individuos de la ontología que representen la unidad médica que hará las solicitudes y la(s) unidad(es) médica(s) que le proveerá(n) la información de pacientes así como el canal de comunicación entre ellas para el intercambio de información.

2. Captura de información necesaria para identificar al paciente desde el cliente.

3. Consulta de la ontología, haciendo uso de Jena, para extraer los elementos necesarios para la construcción del mensaje HL7.

4. Construcción de mensaje HL7 
5. Envío del mensaje, a través de Mirth Connect, utilizando HAPI, hacia el canal descrito en la ontología.

6. Recepción del mensaje de respuesta HL7, interpretando sus campos con ayuda de la ontología para finalmente presentar la información en la pantalla del cliente.

Por otro lado, la funcionalidad de la aplicación proveedor es:

1. Al igual que en la aplicación cliente, es necesario realizar una configuración creando individuos en la ontología que representen la unidad médica que escucha el mensaje, el o los canales de comunicación por donde recibe mensajes, la información del repositorio de la unidad médica (tablas y campos de la base de datos) y conceptos de la ontología que está dispuesto a compartir de acuerdo a la legislación vigente.

2. Recepción de mensaje HL7 procesando los elementos que lo forman.

3. Consulta de la ontología para conocer la equivalencia entre la solicitud HL7 y los elementos específicos del repositorio que serán accedidos.

4. Construcción de consulta SQL para obtener la información del repositorio.

5. Construcción de mensaje HL7 de respuesta obteniendo la equivalencia con ayuda de la ontología.

6. Envío de la respuesta.

\section{Proceso de evaluación de ontologías}

Barchini y Álvarez presentan en [5] varias definiciones de calidad relacionadas:

"La calidad es un conjunto de propiedades y características de un producto o servicio que le confieren su aptitud para satisfacer necesidades explicitas o implícitas

"La calidad de software es el grado con el que un sistema, componente o proceso cumple los requerimientos específicos y las necesidades o expectativas del cliente o usuario".

"La calidad de una ontología es el grado con el que la misma puede ser localizada y recuperada, responde a la estructura invariante del dominio, con un mínimo compromiso ontológico, cumple con los requisitos específicos y puede usarse o reusarse de manera efectiva".

Para realizar la evaluación de ontologías y asegurar la calidad de éstas existen diversos enfoques dependiendo del tipo específico y propósitos de la ontología a evaluar. A continuación se mencionan algunas clasificaciones propuestas por diversos autores.

Barchini y Álvarez [5] también proponen cuatro dimensiones que permiten definir de manera operativa la calidad de una ontología.

1) Descriptiva: referente a brindar información sobre características intrínsecas e identificadoras de la ontología, tal como los desarrolladores de la ontología, los principales destinatarios, los expertos del dominio y los usuarios finales.

2) Estructural: grado en que la ontología especifica de manera explícita, formal y consensuada los conceptos (entidades, atributos, procesos), definiciones, interrelaciones y restricciones del dominio. Dicho de otra manera, corresponde a la sintaxis y semántica formal de la ontología. 
3) Funcional: capacidad de la ontología para proporcionar funciones que satisfagan las necesidades específicas. Permite determinar el grado de concordancia con los requisitos y cuestiones competentes para las que se diseñó.

4) Operacional: capacidad de la ontología para usarse, comunicarse, interactuar e integrarse entre agentes software y/o personas. Esta dimensión refleja la capacidad de uso y reúso que ofrece la ontología.

Gangemi [6] define tres tipos principales de evaluación:

1) Evaluación funcional: se centra en verificar que la ontología cumpla con su objetivo.

2) Evaluación de usabilidad: se ocupa de metadatos y anotaciones.

3) Evaluación estructural: se centra en las propiedades estructurales de la ontología como grafo.

En [7] se menciona que la evaluación de una ontología es mejor si se realiza por niveles en lugar de tratar de evaluarla directamente en conjunto. Los niveles de evaluación se han definido de diversas maneras sobresaliendo los siguientes:

- Léxico de vocabulario o capa de datos: se centra en el vocabulario utilizado para representar o identificar conceptos. La evaluación en este nivel tiende a implicar comparaciones con diversas fuentes de datos relativos al dominio del problema.

- Jerarquía o taxonomía: una ontología incluye típicamente una estructura jerárquica con relaciones tipo "es-un" entre conceptos. Aunque también se definen otro tipo de relaciones entre conceptos, la relación tipo "es-un" es importante y el foco de los esfuerzos específicos de evaluación de este nivel.

- Otras relaciones semánticas: dado que la ontología es posible que contenga otras relaciones además de las tipo "es-un", estas relaciones se evalúan por separado. Esto normalmente incluye medidas como la precisión y la recuperación.

- Nivel de aplicación o contexto: es posible que una ontología forme parte de una colección mayor de ontologías, además de referenciar o ser referenciada por diferentes definiciones dentro de dicho conjunto. En este caso, es importante tener en cuenta este contexto para su evaluación. Otra forma de contexto es la aplicación en la que la ontología se va a utilizar; la evaluación analiza cómo los resultados de la aplicación se ven afectados por el uso de la ontología.

- $\quad$ Nivel sintáctico: la evaluación en este nivel es de particular interés para las ontologías que se han construido, en su mayoría, de forma manual. La ontología está descrita en un lenguaje formal particular y debe coincidir con los requisitos sintácticos de ese lenguaje.

- Estructura, arquitectura y diseño: esta evaluación es de gran interés en ontologías construidas manualmente, donde busca que la ontología cumpla con ciertos principios y criterios de diseño predefinidos. Este tipo de evaluación suele proceder de forma completamente manual.

De acuerdo a [8] la mayoría de los enfoques de evaluación caen en una de las siguientes categorías:

- Basado en la evaluación Estándar Oro: consiste en medir la similitud entre ontologías; existen dos maneras de comparar ontologías: 1) Léxica, que evalúa la similitud entre los léxicos (conjunto de etiquetas que detonan conceptos) y 2) 
Conceptual, donde se comparan las estructuras taxonómicas y las relaciones en las ontologías.

- Basados en el uso de la ontología en una aplicación: donde se evalúa la ontología creando una aplicación que permita comprobar que dicha ontología cumpla con el objetivo para el cual se desarrolló.

- Basados en comparaciones con fuentes de datos: la ontología se evalúa comparando su contenido con fuentes de datos sobre un mismo dominio.

- Basados en metodologías y normas: la evaluación se lleva a cabo por especialistas que tratan de evaluar la eficacia de la ontología, tomando en cuenta un conjunto de criterios predefinidos, normas y requisitos entre otros elementos.

\section{Evaluación de la ontología utilizando OOPS!}

Las aplicaciones que se describen en el apartado anterior permiten verificar la ontología de manera funcional, es decir, validar que cumpla con el objetivo principal para el que se creó, sin embargo no verifican otras condiciones de calidad.

OOPS! es una aplicación Web, independiente de cualquier entorno de desarrollo de ontologías, para la detección de errores de modelado. Esta herramienta está diseñada para ayudar a los desarrolladores de ontologías durante la actividad de validación, misma que se divide en Diagnóstico y Reparación. Actualmente, OOPS! proporciona mecanismos para detectar errores por lo que se ubica en la etapa de Diagnóstico [9].

Es importante mencionar que no todos los problemas encontrados tienen el mismo nivel de importancia, OOPS! maneja tres niveles de resultados.

1. Crítico: Es crucial corregir el problema, de lo contrario podría afectar la consistencia de la ontología, el razonamiento, la aplicabilidad, entre otros.

2. Importante: Aunque el problema no es crítico para la funcionalidad de la ontología, es importante corregir este tipo de problema.

3. Menor: En realidad no es un problema serio, pero la corrección de este tipo de defecto permite que la ontología sea aún más estable.

\subsection{Revisión de la ontología en busca de errores}

En la figura 3 se muestra el código fuente introducido en la aplicación para su evaluación.

\footnotetext{
〈!-- http://www. semanticweb.org/ee/ontologies/2016/4/untitled-ontology-67\#UrgenciaCalificada --〉

〈Class rdf:about="http://www. semanticweb.org/ee/ontologies/2016/4/untitled-ontology-67\#UrgenciaCalificada"〉 $\langle$ rdfs:label rdf:datatype="\&xsd; string" >UrgenciaCalificada〈/rdfs:label〉

〈rdfs: subclassof rdf: resource="http://www. semanticweb.org/ee/ontologies/2016/4/untitled-ontology-67\#Urgencia"/〉 〈disjointwith rdf:resource="http://ww. semanticweb.org/ee/ontologies/2016/4/untitled-ontology67\#UngencialloCalificada"/>

〈rdfs:comment rdf:datatype="\&xsd;string">Problema de salud, habitualmente de presentación súbita, que se atendio en el área de urgencias, que pone en riesgo la vida, órgano, tejido o función del paciente y que, por lo tanto, requiere de una atención médica inmediata. $\langle/ \mathrm{rdf}$ s: comment $\rangle$

$\langle/$ Class $\rangle$
}

Fig. 3. Código fuente de la ontología. 
La aplicación analizó la ontología utilizando Jena, se revisó en busca de problemas de acuerdo a las políticas de OOPS! Durante esta fase de exploración, se detectaron los elementos que intervienen en la ontología y sus posibles errores; además, se generaron advertencias con respecto a la sintaxis de RDF y algunas sugerencias de modelado.

\subsection{Resultado de la evaluación}

El resultado de la evaluación de la ontología se muestra en la figura 4 donde se visualizan cuatro problemas hallados, de los cuales tres son de nivel Menor y uno de nivel Importante. A continuación se describe cada uno de los problemas encontrados.

\begin{tabular}{|c|c|c|}
\hline \multicolumn{2}{|l|}{ Results for P08: Missing annotations. } & 169 cases | Minor \\
\hline Results for P13: Inverse relationships not explicitly declared. & Expand/Collapse & 10 cases | Minor? \\
\hline \multicolumn{2}{|l|}{ Results for P22: Using different naming conventions in the ontology. } & ontology $\mathrm{y}^{\star} \mid$ Minor $\cap$ \\
\hline \multicolumn{2}{|l|}{ Results for P41: No license declared. } & ontology* | Important $\ominus$ \\
\hline
\end{tabular}

Fig. 4. Resultados de la evaluación.

1. Falta de anotaciones: este problema tiene que ver con elementos de la ontología que no cuentan con propiedades de anotación que brinden mayor información al usuario (por ejemplo rdfs:label, lemon:LexicalEntry, skos:prefLabel o skos:altLabel).

2. Falta de relaciones inversas: este problema aparece cuando alguna relación (a excepción de aquéllas que son simétricas, owl:SymmetricProperty) no tiene una relación inversa (owl:inverseOf ) definida dentro de la ontología. Las relaciones inversas son importantes porque facilitan las inferencias y consultas.

3. Uso de diferentes nomenclaturas en la ontología: si bien no existe un estándar de nomenclatura, se sugiere utilizar CamelCase o separación mediante delimitadores (“-” o “_”), además se prefiere utilizar la misma nomenclatura para las mismas cosas y no una mezcla de nomenclaturas.

4. Licencia no declarada: se refiere a la omisión de información acerca de la licencia que se aplica a la ontología.

\subsection{Fase de mantenimiento (Depuración de errores)}

De acuerdo con los resultados de la evaluación de la ontología se procedió a resolver los problemas encontrados. En el primer caso se agregaron anotaciones a cada uno de los elementos de la ontología que no contaban con etiquetado y/o comentario. En la figura 5 se observa que al concepto "AtencionMedica" se le agregaron anotaciones de etiquetado y de comentario. 
E. V. López Hernandez, Beatriz A. Olivares Zepahua, G. S. Hernández Chan, et al.

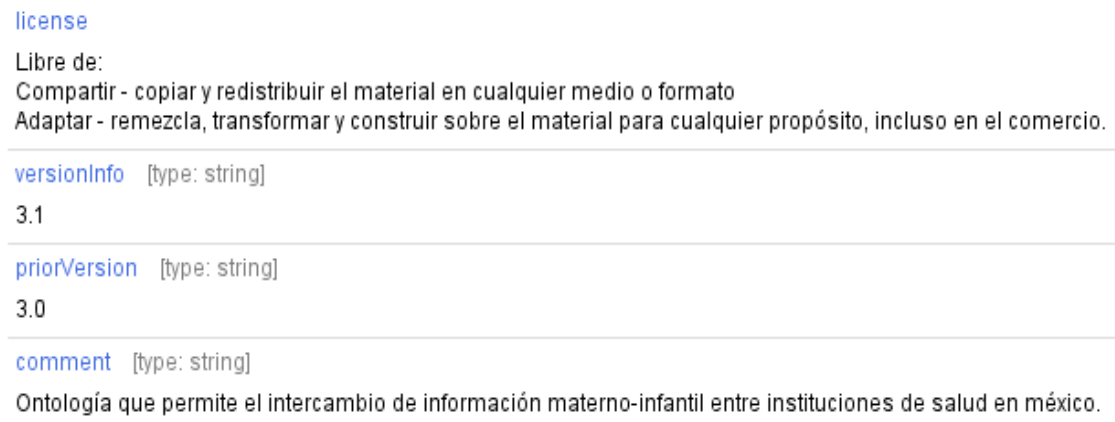

Fig. 7. Información de la licencia, versiones y comentario de la ontología.

\section{Conclusiones $y$ trabajo futuro}

Es sumamente importante contar con herramientas o métodos que permitan la identificación de problemas en el diseño y/o planteamiento de una ontología. Existen muchas formas de evaluación, como las mencionadas en la introducción de este trabajo, muchas de ellas se centran en evaluaciones cuantitativas, sin embargo se encontraron pocas referencias activas a herramientas que realicen evaluación cualitativa, por tal motivo se optó por utilizar la aplicación OOPS! para la detección de errores debido a su naturaleza cualitativa.

OOPS! identifica los errores más comunes cometidos en el planteamiento y desarrollo de una ontología, por lo que ayuda a identificar y solucionar problemas de calidad de una manera más sencilla; los resultados obtenidos en el proceso de evaluación con OOPS! permitieron corregir deficiencias que podrían afectar el correcto funcionamiento de la ontología planteada.

Como trabajo a futuro se implementarán las aplicaciones descritas en el apartado 2 Ontología para el intercambio de información materno-infantil de tal manera que se realice la evaluación funcional de la ontología planteada; como parte de las pruebas se buscará la colaboración de algunas instituciones de salud en México para realizar las validaciones correspondientes.

Agradecimientos. El autor agradece al Consejo Nacional de Ciencia y Tecnología (CONACYT) por el apoyo en el financiamiento del proyecto de Tesis y al Tecnológico Nacional de México por haberlo aceptado y ser parte de él.

\section{Referencias}

1. Sitio Oficial Health Level Seven International. Disponible en: http://www.hl7.org

2. Sitio Oficial Mirth. Disponible en: http://www.mirthcorp.com/products/mirth-connect

3. Sitio Oficial Jena. Disponible en: https://jena.apache.org/documentation/ ontology

4. Sitio Oficial HAPI. Disponible en: http://hl7api.sourceforge.net 
Evaluación de una ontología para el intercambio de información materno-infantil ...

5. Barchini, G. E., Álvarez, M. M.: Dimensiones e indicadores de la calidad de una ontología. Revista Avances en Sistemas e Informática, Disponible en: http://www.redalyc.org/articulo.oa?id=133115523004 (2010)

6. Gangemi, A.: A theoretical framework for ontology evaluation and validation. SWAP, Vol. $166(2005)$

7. Brank, J., Grobelnik, M., Mladenié, D.: A survey of ontology evaluation techniques. In: Proceedings of the conference on data mining and data warehouses (2005)

8. Lu, Q.: OntoKBEval: a support tool for OWL ontology evaluation. Diss. Concordia University (2006)

9. Poveda, M., Suárez, M. C., Gómez, A.: Validating ontologies with Oops! In: International Conference on Knowledge Engineering and Knowledge Management, Springer Berlin Heidelberg (2012) 